\title{
Motion of the hydrogen bond proton in cytosine and the transition between its normal and imino states
}

\author{
Zhen-Min Zhao ${ }^{2}$, Qi-Ren Zhang ${ }^{1,2}$, Chun-Yuan $\mathrm{Gao}^{2}$ and Yi-Zhong Zhuo ${ }^{3}$ \\ ${ }^{1}$ CCAST(World Lab),P.O.Box 8730,Beijing,100080, China \\ ${ }^{2}$ Department of Technical Physics, Peking University, Beijing 100871, China \\ ${ }^{3}$ China Institute of Atomic Energy, Beijing 102413, China
}

\begin{abstract}
The potential energy surface of the H13 proton in base cytosine of the DNA molecules is calculated $a b$ initio at the Gaussian98 MP2/6-311G(d,p) level. Two potential wells are found. One corresponds to the normal cytosine, while the other corresponds to its imino tautomer. The bindings of the proton in these wells are stable enough against the thermo-disturbance. The motions of the proton in these wells are oscillations around the nearest nitrogen atom like the pendula, and may move far away from the nitrogen atom to form the hydrogen bond with other bases. The estimated tunneling probability of the H13 proton from one well to another well shows that the life time of the proton staying in one of these wells is about $6 \times 10^{2} \mathrm{yr}$. It is too long to let tautomers of cytosine be in thermodynamical equilibrium in a room temperature gas phase experiment. The biological significance of these result is discussed.
\end{abstract}

Keywords:Hydrogen bond proton, Potential energy surface, Tunneling

PACS numbers: 87.14.Gg, 87.15.-v

\section{INTRODUCTION}

More than 40 years ago, Per-Olov Lowdin [1] proposed a program for the research of mutation, aging, and cancer formation on the basis of DNA changes due to the tunneling of the hydrogen bond proton through the barrier separating its normal and ill configurations. It was unable to realize this attractive program at that time, since both the theoretical chemical method and the computational ability were not enough developed. Having $a b$ intio method with its fairly developed software package and high performance computers in hand, one is able to try it now. Many works have been done along this direction. References [2]-15] are some earlier examples. However, we do not yet see the previous report on the $a b$ initio calculation about the potential surface and the corresponding motion of the hydrogen bond proton.

The four bases (A, T, C, G) in a DNA molecule usually stay in their normal configurations, but occasionally transfer to their unusual imino/enol configurations with small probabilities. It is the transitions $\mathrm{A} \leftrightarrow \mathrm{A}_{\text {imino }}, \mathrm{T} \leftrightarrow \mathrm{T}_{\text {enol }}, \mathrm{G} \leftrightarrow \mathrm{G}_{\text {enol }}$, and $\mathrm{C} \leftrightarrow \mathrm{C}_{\text {imino }}$. The imino/enol tautomers will be paired in the way $\mathrm{A}_{\text {imino }} \cdots \mathrm{C}, \mathrm{A} \cdots \mathrm{C}_{\text {imino }}, \mathrm{G}_{\mathrm{enol}} \cdots \mathrm{T}$, and $\mathrm{G} \cdots \mathrm{T}_{\mathrm{enol}}$. It makes the possible changes

$$
\mathrm{C} \cdots \mathrm{G} \leftrightarrow \mathrm{T} \cdots \mathrm{A}
$$

of the base pairs in DNA during its double replications. For an example, the transition $\mathrm{C} \rightarrow \mathrm{C}_{\text {imino }}$ changes the pair $\mathrm{C} \cdots \mathrm{G}$ to $\mathrm{C}_{\text {imino }} \mathrm{G}$, and the pairing $\mathrm{A} \cdots \mathrm{C}_{\mathrm{imino}}$ produces pairs $\mathrm{A} \cdots \mathrm{C}_{\mathrm{imino}}$ and $\mathrm{G} \cdots \mathrm{C}$ in a first replication. A further replication produces pairs T...A, $\mathrm{C}_{\text {imino }} \cdots \mathrm{A}, \mathrm{C} \cdots \mathrm{G}$, and $\mathrm{C} \cdots \mathrm{G}$. In the first case, a pair $\mathrm{T} \cdots \mathrm{A}$ appears at the position originally occupied by the pair $\mathrm{C} \cdots \mathrm{G}$. This is a change of heredity code, therefore is serious. It may influence the development and reproduction of a living system, cause aging, cancer, and mutation for examples. To understand and research these kinds of processes at the level of quantitative theoretical physics would be highly desirable.

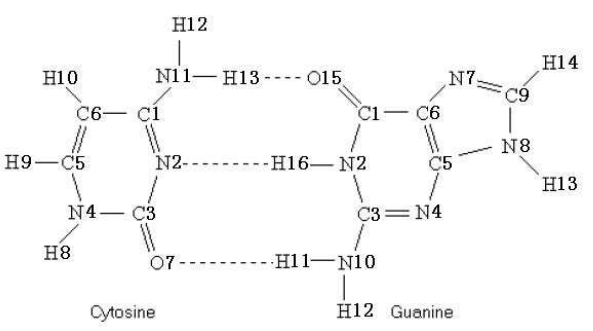

FIG. 1: The bases cytosine and guanine and their pairing by hydrogen bonds (dashed lines) The attached number is for specifying the atom in a base

Figure 1 and 2 show the structure formulae of base cytosine and its imino tautomer, as well as their different pairings. The difference between these two tautomers of cytosine lies mainly in the different positions of the proton H13. This difference in turn causes the different distribution of hydrogen bonds, and finally causes the different pairings. Therefore, to clarify the potential surface of proton $\mathrm{H} 13$ and its motion under the influence of this potential is the key for understanding the transition $\mathrm{C} \rightarrow \mathrm{C}_{\text {imino }}$ between cytosine tautomers and the change $\mathrm{C} \cdots \mathrm{G} \rightarrow \mathrm{T} \cdots \mathrm{A}$ of the base pair in DNA.

Section 2 is devoted to finding the potential surface of the proton H13 (the hydrogen bond proton) for base cytosine by ab initio calculation. In section 3 , we prove 


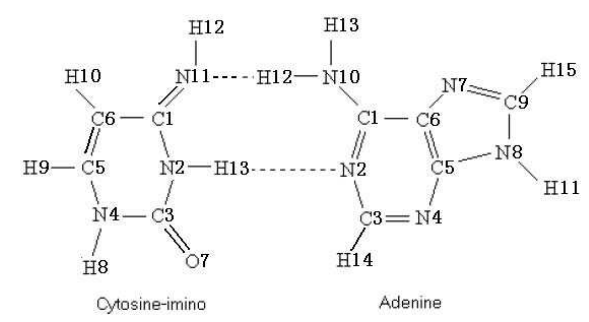

FIG. 2: The bases cytosine-imino and adenine and their pairing by hydrogen bonds

that the proton $\mathrm{H} 13$ is bound in both, normal and imino, configurations, and it is ready for forming the right hydrogen bond of pairing. In section 4 we calculate the tunneling probability of the proton $\mathrm{H} 13$ between these two configurations and discuss its biological significance. Section 5 contains the conclusion and a discussion of the reliability of the results.

\section{POTENTIAL ENERGY SURFACE OF THE PROTON H13 FOR CYTOSINE}

We have calculated the single point energy (SPE) of the proton H13 ab initio by MP2 method in Gaussian98 package with the $6-311 \mathrm{G}(\mathrm{d}, \mathrm{p})$ base set. For every given position of this proton, positions of other atoms in cytosine are optimized by the minimization of the energy of the electron system. It causes a small change of these positions in cytosine. Using a coordinate system fixed on the known structure formula of cytosine, the obtained map of the potential energy surface of the proton H13 is shown in figure 3 . In this figure, the origin of the coordinate system is at the position of N11 in figure 1, the $x$ axis is along its direction $\mathrm{N} 11-\mathrm{C} 1$, and the $y$ axis is perpendicular to $x$ axis and points to the right. The bluer is the point in figure 3 the lower is the potential at that position. The number attached on the line shows the single point energy along the contour. We see clearly two potential wells. The left one corresponds to the cytosine, and the right one corresponds to its imino tautomer. It explains the structure of these two cytosine tautomers shown in figures 1 and 2 .

\section{BINDING OF PROTON H13 IN THE BASE CYTOSINE AND ITS IMINO TAUTOMER}

Around the bottom of a well, we fit the calculated single point energies by a quadratic form of coordinates and find the minimum of SPE by differentiation. The minimum SPE of the left well is -393.9535268 a.u and that of the right well is -393.952654 a.u. Moving the origin of the coordinate system to the position of minimum SPE,

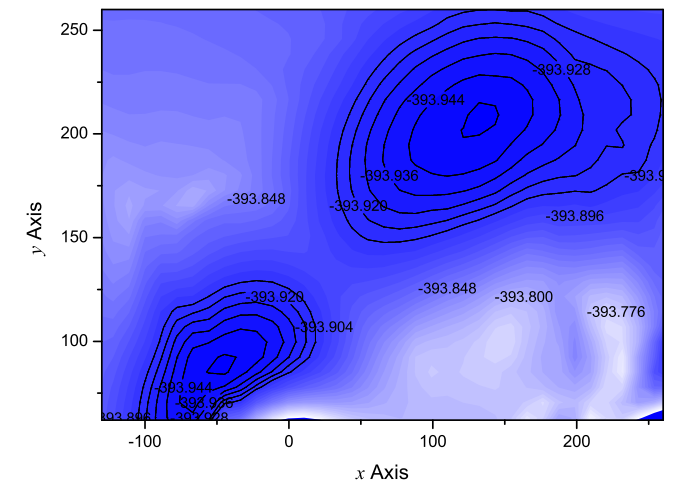

FIG. 3: Contour diagram for the potential energy of the proton $\mathrm{H}(13)$ in cytosine. The energy is in atom unit (a.u.), and the length is in the unit of pico-meter $(\mathrm{pm})$.

the terms containing first degree of coordinate disappear. Finding the principal axes of the coordinate system by the standard method, we may transform the quadratic form into a sum of squares of normal coordinate. For the left well, we take a coordinate system $(\xi, \eta)$ with the $\xi$ axis along the direction N11-H13 in cytosine of figure 1, and $\eta$ axis perpendicular to it. The normal coordinates $(X, Y)$ is related to $(\xi, \eta)$ by a rotation.

$$
\left(\begin{array}{l}
X \\
Y
\end{array}\right)=\left(\begin{array}{rr}
0.94 & -0.34 \\
0.34 & 0.94
\end{array}\right)\left(\begin{array}{l}
\xi \\
\eta
\end{array}\right)
$$

The SPE as a function of normal coordinates $(X, Y)$ is

$$
E=-393.9535+0.088937 Y^{2},
$$

in which the single point energy $E$ is in unit of a.u., and the unit of the length is angstrom. We see that $E$ is independent of the coordinate $X$ at the bottom of this well. The potential surface here looks like a valley along $X$ direction. The transformation (2) shows it is a direction near the line N11-H13. Along this direction, the proton is easier to go far away from the atom N11 to form a hydrogen bond with $\mathrm{O} 15$ in guanine. It explains the pairing shown in figure 1. The oscillation is along the $Y$ direction perpendicular to this direction. It is an oscillation around the atom N11 in cytosine, like a pendulum 'hang' at N11. The frequency is

$$
\begin{aligned}
\nu & =\sqrt{\frac{2 \times 0.088937 \times 27.21 \times 1.60217733 \times 10^{-19}}{1.67231 \times 10^{-27} \times\left(10^{-10}\right)^{2}}} \\
& =3.427 \times 10^{13}(\mathrm{~Hz})
\end{aligned}
$$

and the zero point energy (ZPE) is

$$
\frac{1}{2} h \nu=0.0026 \mathrm{a} \cdot \mathrm{u} \text {. }
$$


Adding this ZPE with the SPE at the bottom of the well, we find that the energy of the proton H13 is -393.9509 a.u.. It is lower than the lowest maximum SPE -393.8895 a.u. around the well. The proton H13 is therefore bound in the normal cytosine, with a binding energy $b=0.0614$ a.u..

For the right well in figure 3 , we take the coordinate system $\left(x^{\prime}, y^{\prime}\right)$, with the $x^{\prime}$ axis parallel the $x$ axis in figure 3 , while its origin is moved to the bottom of the well. The normal coordinates $(X, Y)$ is related to $\left(x^{\prime}\right.$, $\left.y^{\prime}\right)$ by a rotation

$$
\left(\begin{array}{l}
X \\
Y
\end{array}\right)=\left(\begin{array}{rr}
0.30 & -0.95 \\
0.95 & 0.30
\end{array}\right)\left(\begin{array}{l}
x^{\prime} \\
y^{\prime}
\end{array}\right)
$$

The SPE as a function of $X$ and $Y$ is

$$
E=-393.9527+0.12878 Y^{2} .
$$

$E$ is independent of the coordinate $X$ again at the bottom of the well, and the potential surface looks like a valley along the $X$ direction once more. The transformation (6) shows it is a direction almost perpendicular to the line N11-C1. Along this direction, the proton is easier to go far away from the atom N2 of cytosine-imino in figure 2 to form a hydrogen bond with atom N2 in adenine. It explains the pairing shown in figure 2. The oscillation is almost along this line. It is an oscillation around the atom N2 in cytosine-imino, like a pendulum 'hang' at this atom N2. The frequency is

$$
\begin{aligned}
\nu & =\sqrt{\frac{2 \times 0.12878 \times 27.21 \times 1.60217733 \times 10^{-19}}{1.67231 \times 10^{-27} \times\left(10^{-10}\right)^{2}}} \\
& =4.12 \times 10^{13}(\mathrm{~Hz})
\end{aligned}
$$

and the ZPE is

$$
\frac{1}{2} h \nu=0.00313 \mathrm{a} . \mathrm{u} .
$$

The lowest maximum SPE around two wells are at the same point, it is a point between these two wells. The SPE -393.8895 a.u. at this point is called the energy of the transitional state. The sum of the ZPE and the bottom SPE is the energy -393.9496 a.u. of the proton H13 in this well. It is also lower than the transitional state energy. The proton H13 is bound too in the cytosine-imino. The binding energy here is $b^{\prime}=0.0601$ a.u.

The thermo-energy in a living system may be estimated to be $k_{B} T=0.001$ a.u. at the room temperature $T \simeq 300 \mathrm{~K}$. We see that $b$ and $b^{\prime}$ are much larger than the thermal energy. It implies that both cytosine and its imino tautomer are stable against the thermal disturbance.

\section{TUNNELING BETWEEN TWO TAUTOMERS AND ITS BIOLOGICAL SIGNIFICANCE}

According to quantum mechanics, there is still a possibility of the proton tunneling from one well to another well. We first find the path of lowest potential connecting the centers of these two wells. It is described by the equation

$$
\begin{aligned}
y(x) & =1.1417352+0.71244583 x+0.3508047 x^{2} \\
& +0.04422011 x^{3}+0.06419875 x^{4}-0.56534255 x^{5} \\
& +0.25248558 x^{6}
\end{aligned}
$$

in the coordinate system used in figure 3 , with the length unit being changed to angstrom. The potential energy in atom unit along the path is fitted by the formula

$$
\begin{aligned}
V(x) & =-393.908877+0.103691 x-0.102525 x^{2} \\
& -0.3063312 x^{3}+0.102250 x^{4}+0.355250 x^{5} \\
& -0.195806 x^{6}
\end{aligned}
$$

The potential barrier between two wells of the proton H13 is shown in Figure 4. According to the WKB formula, the penetration probability of the proton through this barrier is

$$
\begin{aligned}
W & =\exp \left\{-\frac{2}{\hbar} \int_{s} \sqrt{2 m_{p}[V(x)-E]} \mathrm{d} l\right\} \\
& =\exp \left\{-\frac{2}{\hbar} \int_{a}^{b} \sqrt{2 m_{p}[V(x)-E]\left[1+\left(\frac{\mathrm{d} y}{\mathrm{~d} x}\right)^{2}\right]} \mathrm{d} x\right\} \\
& \approx \exp [-54.85] \approx 1.50929 \times 10^{-24} .
\end{aligned}
$$

Here $E=-393.9496$ a.u. is the total energy of the proton H13 in cytosine-imino, $a$ and $b$ are the $x$ coordinates of the proton to be determined by the condition $E=V(x)$ with $b>a$, and $s$ is the lowest potential path terminated by points $x=a$ and $x=b$.

The penetration probability in unit time is

$$
\begin{aligned}
P & =\nu W=3.427 \times 10^{13} \times 1.50929 \times 10^{-24} \mathrm{~Hz} \\
& =5.168 \times 10^{-11} \mathrm{~s}^{-1}
\end{aligned}
$$

Therefore, the mean life time for a proton staying in one of these wells is $6 \times 10^{2}$ years. It means the time needed for reaching the thermo-equilibrium is more than six hundred years. One could not see this equilibrium of gas type cytosine and its imino tautomer in his one hundred year life. We had better to assume that the large ratio between populations of cytosine and its imino tautomer in nature is not a result of thermo-equilibrium, but rather the natural selection resulting in biological evolution. From (13) we also see, in one's one hundred year life time, about sixteen percent of his cytosine bases may have spontaneously transferred into some imino form. Some of them may transfer back into normal form of cytosine, while 


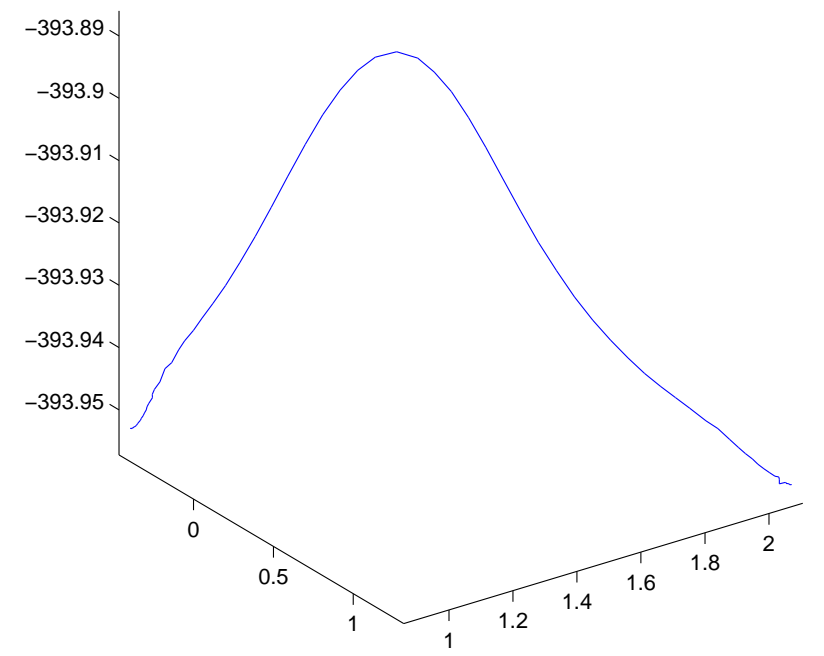

FIG. 4: The potential barrier between two wells of the H13 proton

some others, according to Lowdin's conjecture, may induce aging, mutation or cancer in further replications of DNA. Of course, cytosine as well as other bases of DNA molecules are not isolated in living systems. Their transitions to 'ill' tautomers must be influenced by their environment. To clarify this influence, one has to do more complicated calculations for a base-environment system. A deeper research along this direction would be interesting.

\section{CONCLUSION AND DISCUSSION}

The $a b$ initio quantum mechanical calculation reproduces the structures of cytosine and its imino tautomer, including the possible positions and directions of their hydrogen bond formation. It makes us be confident that this method is applicable and effective in the bio-physics research, and predict the transition probability between normal and imino states of cytosine. The result is reasonable. Its biological significance is discussed above and worthy to explore further.

There are a large number of atoms in a bio-molecule. The energy of the molecule is contributed by atoms in it and by their interactions. The energy change of the molecule in the physical, chemical or biological reactions is usually a tiny part of the total energy of the molecule. To study the behavior and the energy change of the biomolecule in these reactions, we need very precise calculations to keep many digits of the value be correct. For an example, as we have seen at the beginning of section 3 , to compare the single point energy of the proton at the centers of two wells, we need at least first 6 digits of the calculated values be precise. This is not easy in such a huge amount of numerical calculations. For the results reported here, the general tendency of the potential surface may be reliable, but the difference of SPE for specially chosen pair of points may not be so, since the first 5 most reliable digits are subtracted. On the other hand, the tunneling probability may be reliable, since a part of statistical errors may be canceled by each other in an integration. Anyway, to develop the more precise programs with specified upper bound of possible errors would be very desirable in further researches along this direction.

This Work was supported by National Nature Science Foundation Committee of China with grant numbers 10305001 and 10475008, and by the High Performance Computing Center of China (Beijing).
[1] Per-Olov Lowdin, Rev. Mod. Phys. 35 (1963) 724.

[2] J. W. Drake, E. F. Allens, S. A. Forsberg, R. M. Preparata and E. O. Greening, Nature 221 (1969) 1128.

[3] R. V. Wolfernen, J. Mol. Biol. 40 (1969) 307.

[4] M. J. Nowak, K. Szczepaniak, A. Barski and D. Shrgar, Z. Natreforsch C 33 (1978) 876.

[5] M. Raszka, and N. O. Kaplan, Proc. Nat. Acad. Sci. USA 69 (1972) 2025.

[6] R. R. Shoup, H. T. Miles and E. D. Becker, J Phys Chem. 76 (1972) 64.

[7] M. D. Topal, J. R. Fresco, Nature (London) 263 (1976) 285.

[8] M. D. Topal, J. R. Fresco, Nature (London) 263 (1976) 289.

[9] W. Saenger, Principles of Nucleic Acid Structure
(Springer-Verlag, New York, 1984).

[10] R. Rein and F. E. Harris, J. Chem. Phys. 43 (1965) 4415.

[11] C. Colominas, F. J. Luque, M. Orozco, J. Am. Chem. Soc. 118 (1996) 6811.

[12] C. N. R. Rao, Jerus. Symp. Quant. Chem. Biochem. 5 (1973) 107.

[13] M. Geller and B. Lesyng, Biochem. Biophys. Acta 417 (1975) 407.

[14] I. Kulakowska, M. Geller, B. Lesyng, K. Bolewska, and K. L. Wierzchowski, Biochem. Biophys. Acta 417, (1975) 420.

[15] K. G. Rao and C. N. R. Rao, J. Chem. Soc. Perkin Trans. 2 (1973) 889. 